\title{
ON DIFFERENTIABLE FUNCTIONS HAVING AN EVERY WHERE DENSE SET OF INTERVALS OF CONSTANCY
}

A. M. Bruckner ${ }^{(1)}$ and John L. Leonard ${ }^{(2)}$

(received April 10, 1964)

The Cantor function C [2; p. 213], which appears in analysis as a simple example of a continuous increasing function which is not absolutely continuous, has the following properties:

(i) C is defined on $[0,1]$, with $\mathrm{C}(0)=0, \mathrm{C}(1)=1$;

(ii) $\mathrm{C}$ is continuous and non-decreasing on $[0,1]$;

(iii) $\mathrm{C}$ is constant on each interval contiguous to the perfect Cantor set $P$;

(iv) $C$ fails to be constant on any open interval containing points of $P$;

(v) The set of points at which $C$ is non-differentiable is non-denumerable.

Furthermore, it is impossible to "smoothen" C; more precisely it is impossible to modify the function $C$ to a differentiable function satisfying conditions (i) through (iv).

This result will follow from the theorem below.

On the other hand, there do exist everywhere differentiable non-constant functions having intervals of constancy whose union

(1) The work of this author was supported by NSF Grant GP 1592.

(2)

NSF Cooperative Graduate Fellow.

Canad. Math. Bull. vol. 8, no. 1, February 1965 
is dense in $[0,1]$. For a construction of such a function see Zahorski[5]. A natural question to ask is: For what nowhere dense perfect sets $P([0,1]$ are there differentiable functions which are constant on each interval contiguous to $\mathrm{P}$, but not constant on any open interval containing points of $\mathrm{P}$ ?

Zahorski [4; p. 43] solves the broader problem of characterizing the set $E$ of zeroes of a bounded derivative. He gives as a necessary and sufficient condition that $\sim E$ be an $F_{\sigma}$ satisfying a complicated density condition which he calls $\mathrm{M}_{4}$. We do not define $\mathrm{M}_{4}$, but simply mention that the condition $\mathrm{M}_{4}$ is implied by the stronger but simpler condition $M_{5}$, that each point of the $F_{\sigma}$ set $A$ is a point of density of $A$. Zahorski notes that his result characterizes the set of zeroes of the bounded derivative of a function having intervals of constancy whose union is dense. However, a precise knowledge of the set of zeroes is superfluous to the question we ask, and, therefore, a condition simpler than $\mathrm{M}_{4}$ can provide our answer.

THEOREM. Let $P$ be a nowhere dense perfect subset of $[0,1]$ and let $G=\sim P$. A necessary and sufficient condition that there exist a differentiable function $f$ which is constant on each component interval of $G$, but not constant on any open interval containing points of $P$, is that the intersection of $P$ with any open interval is either empty or has positive measure.

Proof: Necessity. Let $P$ be any perfect set having nonempty zero measure intersection with some open interval $I$. If $f$ is differentiable on $I \cap[0,1]$ then $f$ satisfies Lusin's condition $(N)$, that is, if $Z$ is a null subset in the domain of $f$, then the set $f[Z]$ is also a null set. (See Saks, [3, p. 271].) Thus $f[P \cap I]$ is a null set. Now, if $f$ is constant on each interval contiguous to $P$, then $f[I \sim P]$ is at most countable. It follows that $f[I]$ is a null set. Since $f$ is continuous, $f$ must be constant on I. But I contains points of $P$, so $f$ does not meet the requirements of the theorem. This proves the necessity of the condition.

Sufficiency. Let $P$ be a perfect set whose intersection with every open interval is either empty or has positive measure. 
Let $D *$ be the set of points of density of $P$ and let $D=D * \cap P$. Since almost all points of $P$ are points of density of $P$ we have $\mathrm{mD}=\mathrm{mP}$, where $\mathrm{m}$ denotes Lebesque measure. Let $E$ be an $F_{\sigma}$ contained in $D$ such that $m E=m D$. Ther every point of $E$ is a point of density of $E$. It follows from the result of Zahorski cited above that there is a differentiable function $f$ whose derivative vanishes exactly on $\sim E$. If $I$ is any interval contiguous to $P$ then $f^{\prime}=0$ on $I$, so $f$ is constant on $I$.

On the other hand, since $E$ is dense in $P$, any open interval I which contains points of $P$ also contains points of $E$, and thus $f^{\prime}$ does not identically vanish on $I$. Thus the sufficiency is proven.

We conclude with two remarks:

1. Part of the proof of the sufficiency of the condicion is similar to a proof of a related theorem due to Boboc and Marcus [1]. They proved that a necessary and sufficient condition that a set $E C[0,1]$ be determining for the class of bounded derivatives is that the complement of $E$ have interior measure zero. (A set $E$ is determining for a class of functions $C$ if whenever $f=g$ on $E$ and $f, g \in C$, then $f \equiv g$.)

2. It is worth noting that if a differentiable function $f$ is constant on each interval contiguous to the nowhere dense perfect set $P$, then $f^{\prime}$ must vanish at uncountably many points of $P$ a s well as at every point of $\sim P$. This follows from the fact that the set at which $f^{\prime}$ does not vanish is an $F_{\sigma}$.

\section{REFERENCES}

1. N. Boboc and S. Marcus, Sur la détermination d'une fonction par les valeurs prises sur un certain ensemble, Ann. L'Ecole Norm. Sup. (3), 76, (1959), 151-159.

2. I. P. Natanson, Theory of functions of a real variable, Vol. 1, Ungar, New York, 1961.

3. S. Saks, Theory of the integral, Monografje Matematyczne, Vol. 7, Warszawa - Lwów, 1937. 
4. Z. Zahorski, Sur la première dérivée, Trans. Amer. Math. Soc. 69 (1950), 1-54.

5. , Über die Konstruktion einer differenzierbaren, monotonen, nicht Konstanten Funktion, mit überall dichter Menge von Konstanzintervallen, Comptes Rendus des Séances de la Société des Sciences et des Lettres de Varsorie, classe III, vol 30 (1937), 202-206.

University of California,

Santa Barbara 